\title{
Clinical and Radiological Dissociation in Massive Barium Aspiration
}

\author{
Ramakanth Pata ${ }^{1}$, Tsering Dolkar ${ }^{1}$, Meet J. Patel ${ }^{1}$, Nway Nway ${ }^{1}$, Htun M. Aung ${ }^{1}$ \\ 1. Internal Medicine, Interfaith Medical Center, Brooklyn, USA
}

Corresponding author: Ramakanth Pata, cookybrey1@gmail.com

\begin{abstract}
Barium studies are commonly used to rule out gastrointestinal (GI) pathologies and sometimes they are associated with complications such as barium aspiration with heterogeneity in clinical features ranging from mild to severe symptoms. We present a case of large volume barium aspiration in a 73-year-old male with past medical history of dysphagia diagnosed with diffuse esophageal spasm. Barium is an inert material commonly used for GI tract study. Although complications associated with barium studies are rare, aspiration of barium can have dramatic findings resulting in mild to severe symptoms. Clinically patient had very minimal symptoms but radiographic studies appeared dramatic. Therefore, a clinical and radiographic paradox must be kept in mind when evaluating patients and reviewing large volume barium aspiration imaging. Our case remained asymptomatic and had no respiratory complaints, nor did he develop any respiratory distress post barium aspiration.
\end{abstract}

Categories: Radiology, Gastroenterology, Pulmonology

Keywords: barium aspiration

\section{Introduction}

Barium is an inert material commonly used for gastrointestinal (GI) tract study. We present a case of large volume barium aspiration in a 73-year-old male with past medical history of dysphagia diagnosed with diffuse esophageal spasm. Although complications associated with barium studies are rare, aspiration of barium can have dramatic findings resulting in mild to severe symptoms. Clinically patient had very minimal symptoms but radiographic studies appeared dramatic.

Review began 12/24/2020 Review ended 01/06/2021 Published 01/07/2021

\section{๑) Copyright 2021}

Pata et al. This is an open access article distributed under the terms of the Creative Commons Attribution License CC-BY 4.0., which permits unrestricted use, distribution, and reproduction in any medium, provided the original author and source are credited.

\section{Case Presentation}

A 73-year-old male presented to emergency department due to presyncope with an episode of loss of consciousness for three minutes. He also complained of productive cough with whitish sputum. Vital signs were normal and physical examination was benign. Chest X-ray (CXR) showed evidence of consolidation, which was followed by a computed tomography (CT) chest that revealed a dilated esophagus (Figure 1). Barium swallow was done for optimal evaluation of dilated esophagus (Figure 2). Post-procedure CXR revealed interval development of complete opacification of left hemithorax and retained barium in the left upper chest (Figure 3). CT chest was followed, which revealed retained barium within the upper to the midthoracic esophagus. Complete left lung atelectasis was noted, likely related to the obstructing filling defect within the left main bronchus (Figure 4). Subsequently, the CXR showed persistently retained contrast within the esophagus and improved left lobar airspace disease with moderate to large pleural effusion (Figure 5). However, bedside sonogram showed predominantly consolidation. The patient had no symptoms and was clinically stable to be discharged. He was advised to follow up as an outpatient at the chest clinic in six weeks. 


\section{Cureus}

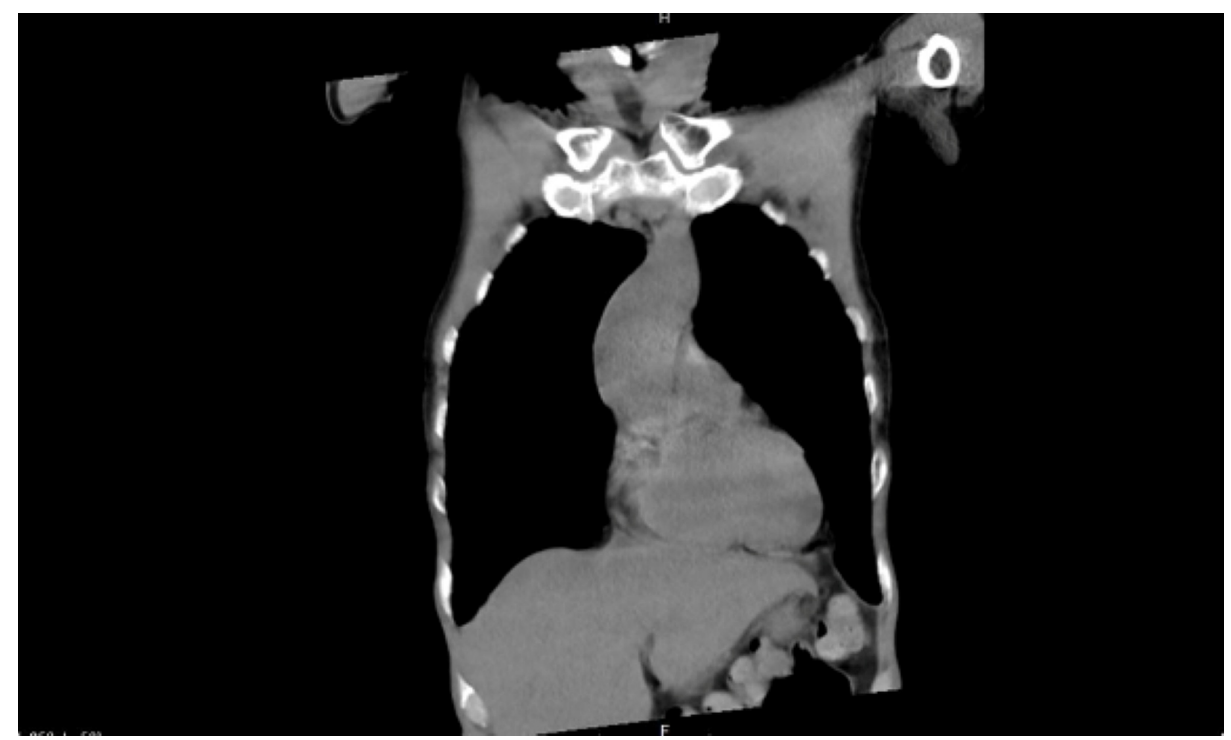

FIGURE 1: CT chest without contrast revealed fluid-filled dilated esophagus, which was suboptimally evaluated due to lack of contrast. The patient also had left lower lobe consolidation consistent with pneumonia.

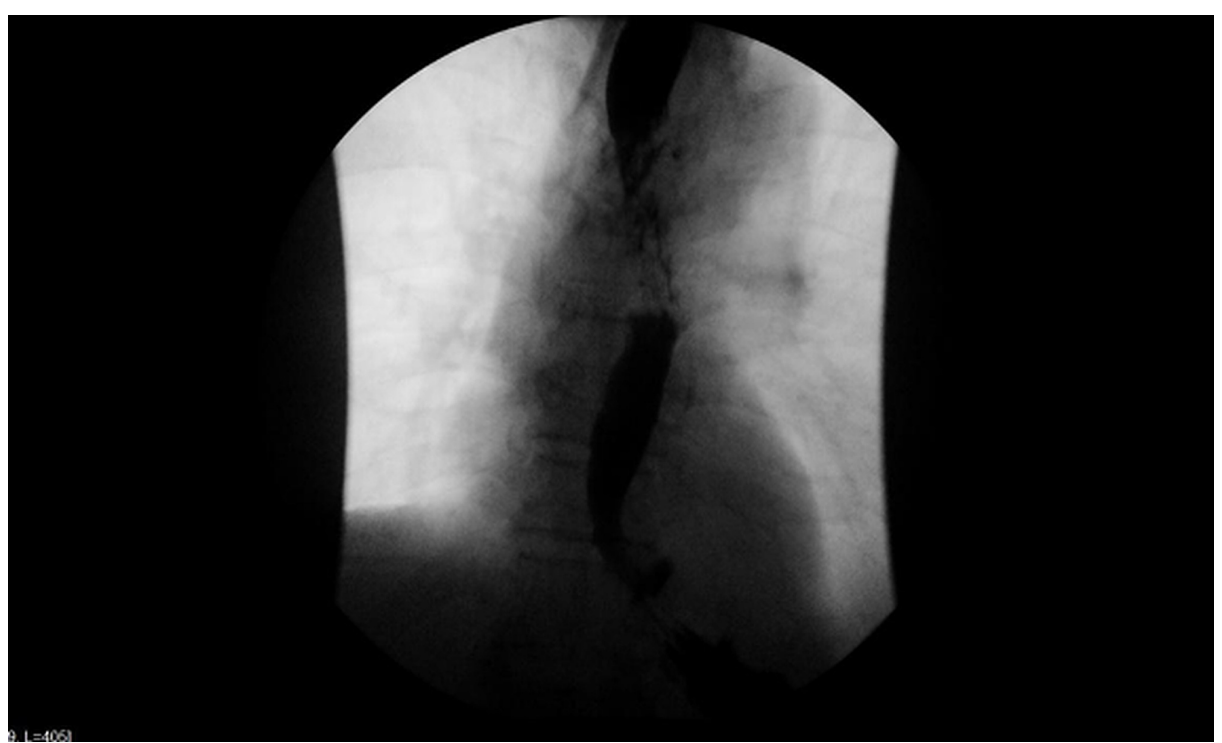

FIGURE 2: A barium study was done, which revealed diffuse esophageal spasm and akinesis of the mid-thoracic esophagus. 


\section{Cureus}

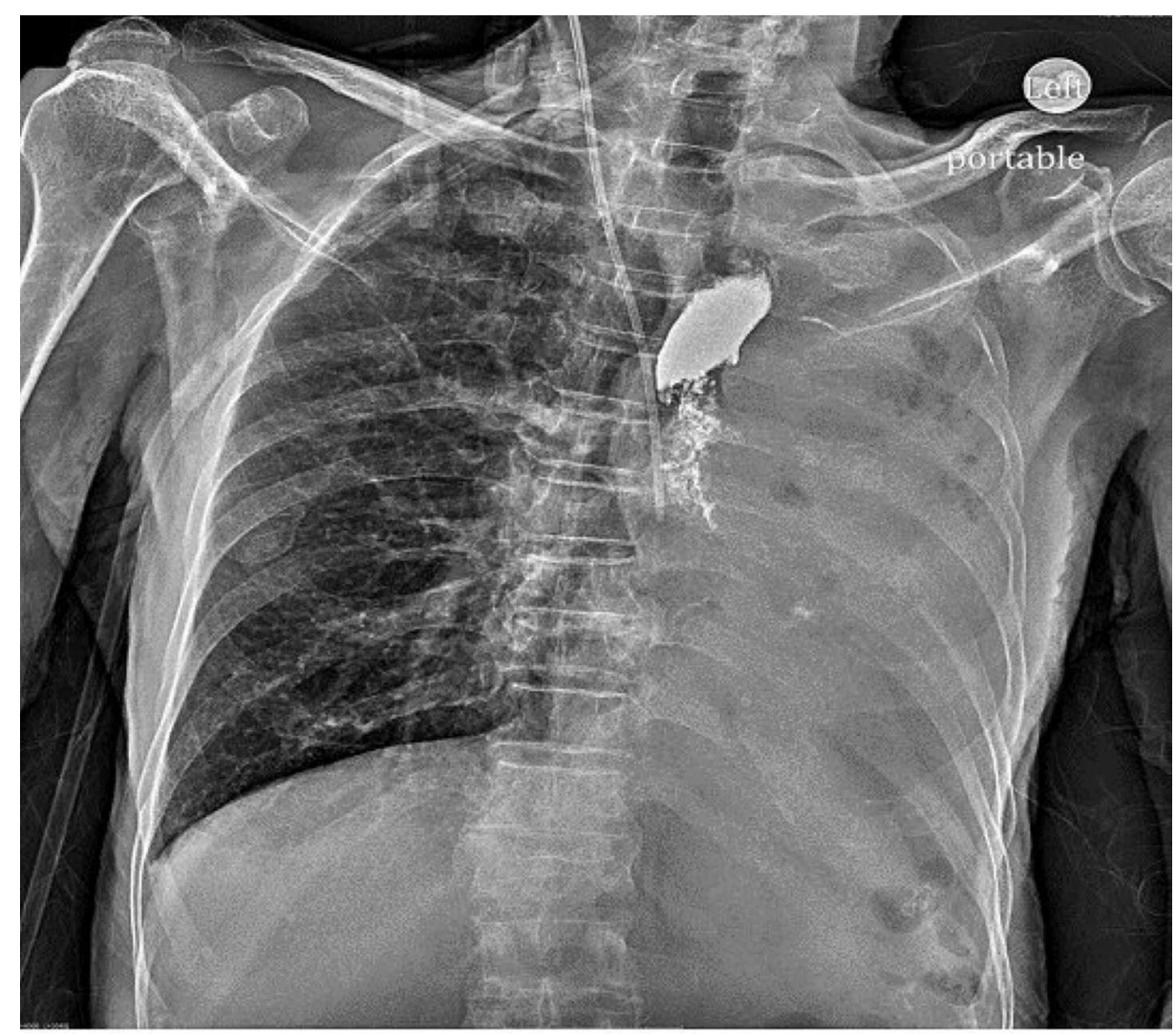

FIGURE 3: Chest X-ray revealed interval development of complete opacification of left hemithorax and retained barium in the left upper chest.

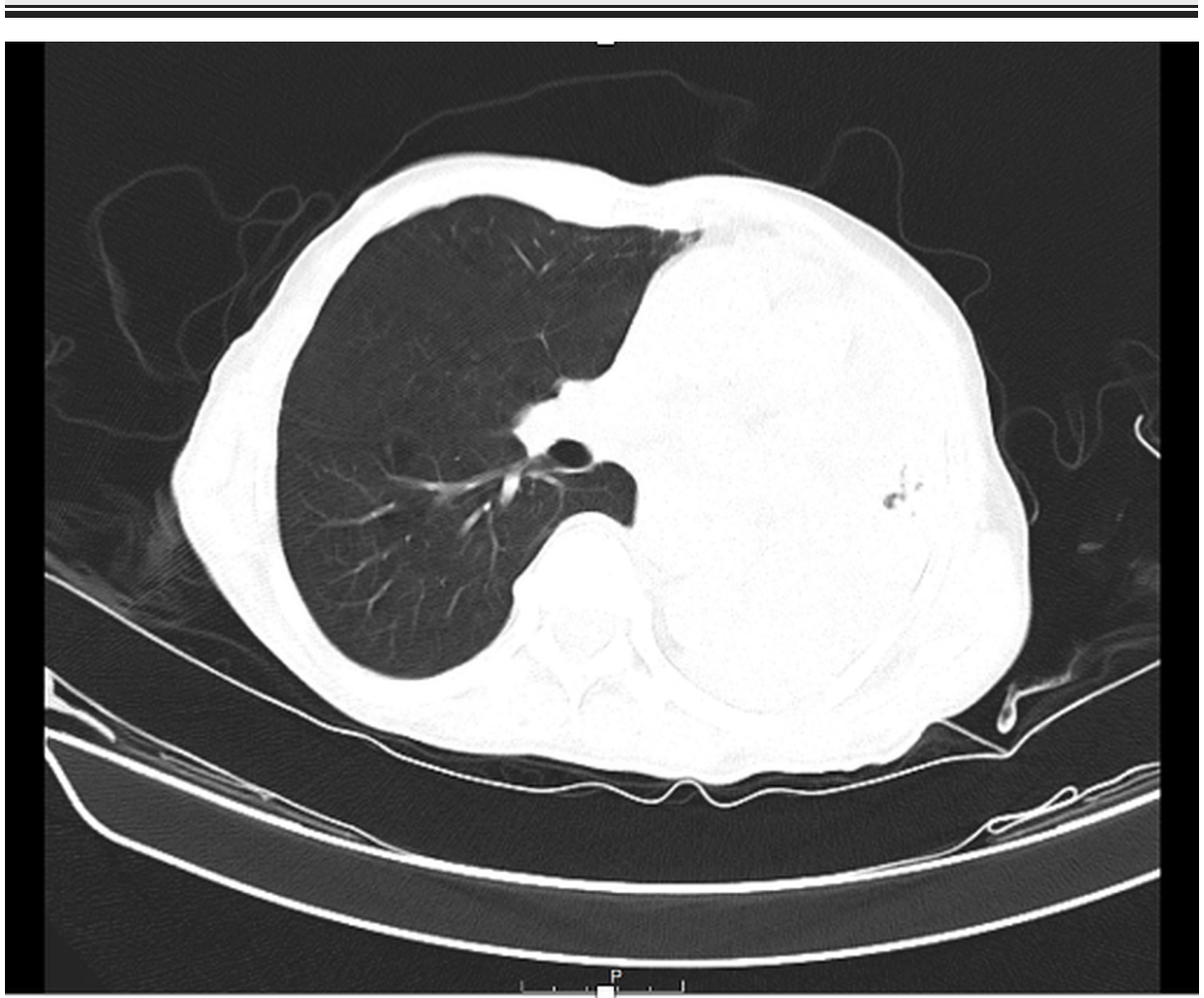

FIGURE 4: CT chest revealed retained barium within the upper to the mid-thoracic esophagus. Complete left lung atelectasis was noted, likely related to the obstructing filling defect within the left main 


\section{Cureus}

bronchus.

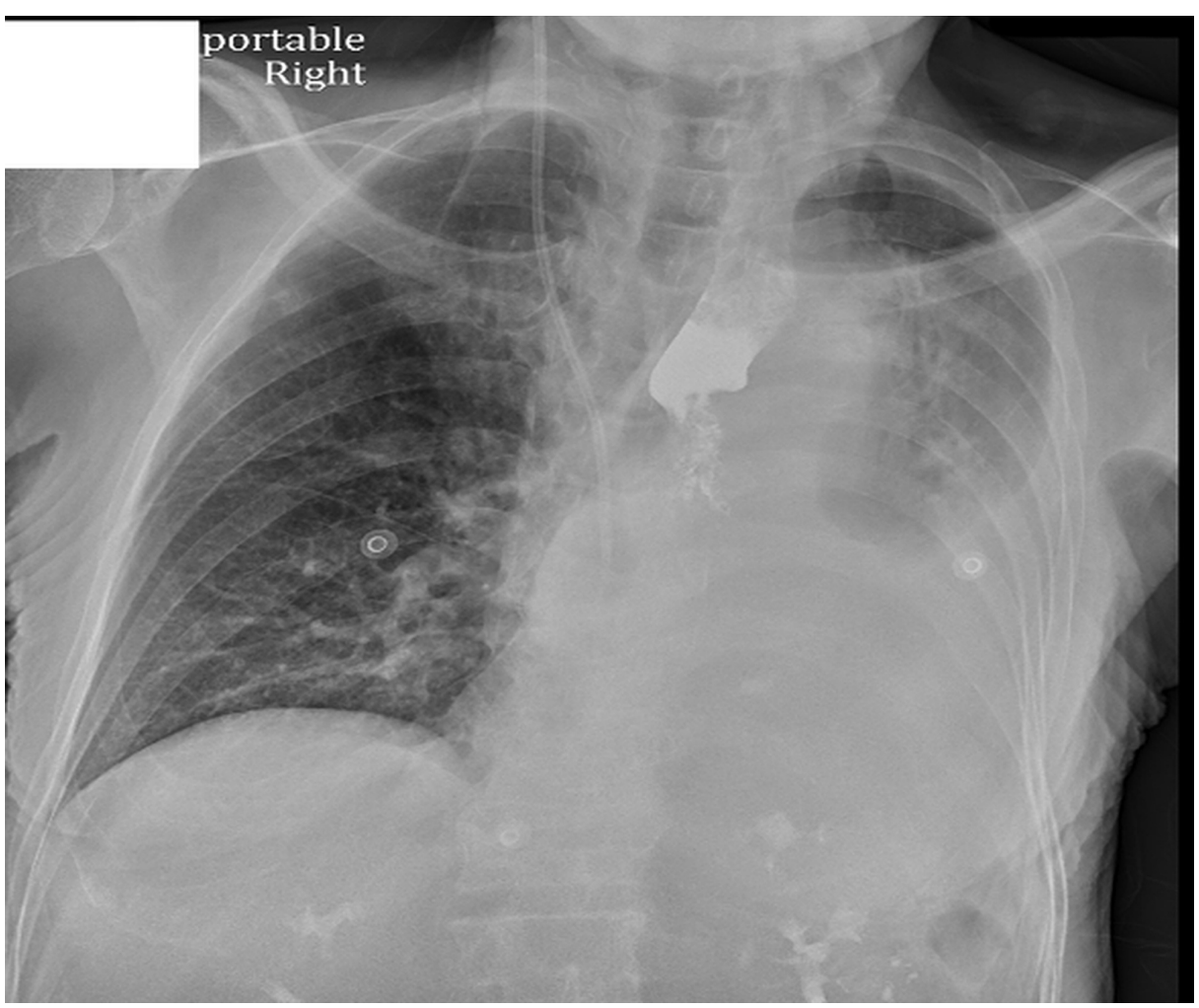

FIGURE 5: Subsequently, a chest x-ray showed persistently retained contrast within the esophagus and improved left lobar airspace disease with moderate to large pleural effusion.

\section{Discussion}

Barium studies are regularly performed to rule out GI lumen pathologies. Despite the risk of barium aspiration, which is present, the complications are rare [1,2]. We present a case of large volume barium aspiration which was clinically asymptomatic with dramatic radiographic findings. In most cases, patients remain largely asymptomatic despite the paradoxical radiographic findings [3]. However, mechanical obstruction leading to atelectasis as well as uncommon acute inflammatory reactions have been reported [35].

There are few articles published reporting mild cases of large volume barium aspiration [1]. Most patients do not develop severe symptoms as seen on the imaging, which is alarming. Our case remained asymptomatic and neither had any respiratory complaints nor did he develop any respiratory distress post barium aspiration. CT chest revealed complete left lung atelectasis, which subsequently improved within days, revealing moderate pleural effusion. Although there was a pleural effusion on the CXR, a bedside sonogram of the lung revealed a predominant consolidation pattern. The patient was kept under observation. A 6minute walk test was carried out and no desaturation or symptoms were noted. Steroids or antibiotics were not used and bronchoscopy was not performed due to the risk of spreading the contrast to unaffected areas [4]. The patient was asked to follow up in the pulmonary clinic six weeks post-discharge.

We prefer to classify patients based on radiographic, physiologic, and clinical parameters: positive radiographic findings and no change in physiological findings; positive radiographic findings and altered ventilation/perfusion (V/Q) ratio [6]; positive radiographic findings with shunt effect [7]; and positive radiographic findings with inflammatory response [8]. Most patients are completely asymptomatic after aspiration and treatment remains observation [2]. Aspiration pneumonia should be suspected whenever a patient complains of dysphagia and radiographic evidence of consolidation. Nevertheless, the risk of aspirations and complications remains high in patients with dysphagia or the elderly [1,9]. Treatment strategies vary depending on the underlying baseline comorbidities in patients with severe respiratory complications, although bronchoscopy and suctioning have been tried [10]. Furthermore, if gastric contents are aspirated along with barium, antibiotics have been used [11]. 
Most patients appear to recover completely one-year post aspiration. High-resolution CT has reported subtle evidence of early fibrosis even one-year post aspiration, although limited data is available on patient outcome years after aspiration [3]. The clinical significance of such findings remains unknown.

\section{Conclusions}

Although barium aspiration has dramatic radiographic findings, most patients are asymptomatic. Therefore, a clinical and radiographic paradox must be kept in mind when evaluating the patients and reviewing large volume barium aspiration imaging. We recommend longer follow-ups to check for delayed developments post barium aspiration.

\section{Additional Information}

\section{Disclosures}

Human subjects: Consent was obtained by all participants in this study. Conflicts of interest: In compliance with the ICMJE uniform disclosure form, all authors declare the following: Payment/services info: All authors have declared that no financial support was received from any organization for the submitted work. Financial relationships: All authors have declared that they have no financial relationships at present or within the previous three years with any organizations that might have an interest in the submitted work. Other relationships: All authors have declared that there are no other relationships or activities that could appear to have influenced the submitted work.

\section{References}

1. Hundemer G, Kumar V, Vaduganathan M: Large-volume barium aspiration. Proc (Bayl Univ Med Cent). 2015, 28:183-184. 10.1080/08998280.2015.11929222

2. Varatharaj A, Roome C, Allsup S: Barium aspiration. QJM. 2012, 105:903-904. 10.1093/qjmed/hcr146

3. Shook CD, Felson B: Inhalation bronchography. Chest. 1970, 58:333-337. 10.1378/chest.58.4.333

4. Tamm I, Kortsik C: Severe barium sulfate aspiration into the lung: clinical presentation, prognosis, and therapy. Respiration. 1999, 66:81-84. 10.1159/000029344

5. Gray C, Sivaloganathan S, Simpkins KC: Aspiration of high-density barium contrast medium causing acute pulmonary inflammation-report of two fatal cases in elderly women with disordered swallowing. Clin Radiol. 1989, 40:397-400. 10.1016/S0009-9260(89)80135-7

6. Kumar A, Kumar A, Kumar N, Sinha C, Singh J: Barium sulfate aspiration: is early bronchioalveolar lavage a life-saving procedure?. Turk J Emerg Med. 2020, 20:50. 10.4103/2452-2473.276383

7. Chiu CY, Wong KS, Tsai MH: Massive aspiration of barium sulfate during an upper gastrointestinal examination in a child with dysphagia. Int J Pediatr Otorhinolaryngol. 2005, 69:541-544. 10.1016/j.ijporl.2004.11.008

8. Gray C, Sivaloganathan S, Simpkins CK: Aspiration of high-density barium contrast medium causing acute pulmonary inflammation: report of two fatal cases in elderly women with disordered swallowing. Clin Radiol. 1989, 40:397-400. 10.1016/S0009-9260(89)80135-7

9. Voloudaki A, Ergazakis N, Gourtsoyiannis N: Late changes in barium sulfate aspiration: HRCT features . Eur Radiol. 2003, 13:2226-2229.

10. Fruchter O, Dragu R: Images in clinical medicine. A deadly examination . N Engl J Med. 2003, 348:1016. 10.1056/NEJMicm020803

11. Kaira K, Takise A, Goto T, Horie T, Mori M: Barium sulfate aspiration. Lancet. 2004, 364:2220. 10.1016/S0140-6736(04)17595-8 